www.jmscr.igmpublication.org

Impact Factor 3.79

Index Copernicus Value: 5.88

ISSN (e)-2347-176x ISSN (p) 2455-0450

crossref DOI: http://dx.doi.org/10.18535/jmscr/v4i1.24

Journal Of Medical Science And Clinical Research

\title{
Atheroembolic Renal Disease (AERD)
}

\section{Authors \\ Lakshminarayana GR ${ }^{1}$, Sheetal LG ${ }^{2}$, Seethalekshmy NV ${ }^{3}$, Anil $\mathbf{M}^{4}$, Rajesh $\mathbf{R}^{5}$, George $\mathbf{K}^{6}$, Unni VN $\mathbf{N}^{7}$}

${ }^{1}$ Consultant Nephrologist, Dept of Nephrology, EMS Memorial Cooperative Hospital and Research Centre, Perinthalmanna, Malappuram, Kerala

${ }^{2}$ Associate Professor, Dept of Physiology, MES Medical College, Perinthalmanna, Malappuram, Kerala,

${ }^{3}$ Professor, Dept of Pathology, Amrita Institute of Medical Sciences and Research Centre, Kochi, Kerala

${ }^{4}$ Associate Professor, Department of Nephrology, Amrita Institute of Medical Sciences and Research Centre, Kochi, Kerala

${ }^{5}$ Professor, Dept of Nephrology, Amrita Institute of Medical Sciences and Research Centre, Kochi, Kerala

${ }^{6}$ Professor and Head, Dept of Nephrology, Amrita Institute of Medical Sciences and Research Centre, Kochi, Kerala

${ }^{7}$ Senior Consultant, Nephrology, CoE Nephrology and Urology, Aster Medicity, Kochi, Kerala

Corresponding Author

\section{Dr Lakshminarayana GR}

Consultant Nephrologist, Department of Nephrology, EMS Memorial Cooperative Hospital and Research Centre, Perinthalmanna, Malappuram, Kerala, India-679322

Email:drlng23@gmail.com, Phone: (+91)9495161833

\section{ABSTRACT}

Background: The atheroembolic renal disease (AERD) is part of multisystem disease is characterised by as renal failure and evidence of peripheral cholesterol embolization. There is no data regarding AERD from India except for few case reports. The present study is the first of its kind from India to report on AERD.

Materials and methods: All the patients who were referred to Nephrology unit at Amrita Institute of Medical Sciences and Research Centre, Kochi, Kerala and diagnosis as AERD based on clinical presentation and/or biopsy, were included in the study.

Results: There were 11 cases of renal failure (males:10; female:1), aged 55-75 years (Mean: 60; SD:9.5 years) due to AERD, with varying precipitating factors. Majority (9 out of 11) of the patients had precipitating factors for atheroembolization. Majority (10 out of 11) of the patients had severe acute renal failure $(A R F)(e G F R<30 \mathrm{ml} / \mathrm{min} / 1.73 \mathrm{~m} 2)$ requiring dialysis therapy. All those requiring renal replacement therapy $(R R T)$ were treated with hemodialysis. Of the 10 patients who required hemodialysis only 4 patients could discontinue hemodialysis after 2 weeks to 6 months and rest were continuing hemodialysis at the time of study. The patient, who did not need dialysis, had partial improvement in renal function with conservative treatment.

Conclusions: The AERD is one the causes for renal failure in elderly patients undergoing vascular intervention procedures and thrombolysis, especially if they have underlying atherosclerotic vascular disease. Majority of the affected patients are males of elderly age and have precipitating factors for atheroembolization in form of coronary interventions, thrombolysis or intake of antiplatelet agents. Majority 
of the presented with acute renal failure. Majority of patients with AERD require renal replacement therapy $(R R T)$ and have poor chance of renal recovery.

Keywords: Atheroembolic renal disease, rapidly progressive renal failure, thrombolysis, coronary angiography, anticoagulation.

\section{INTRODUCTION}

The atheroembolic renal disease (AERD) is characterised by as renal failure secondary to the occlusion of renal arteries, arterioles, and glomerular capillaries with atheromatous plaques that are dislodged from the aorta and other major arteries. [1] [2] Atheroembolic renal disease (AERD) is characterized by the presence of a triad: 1) a precipitating event, 2) subacute renal failure, and 3) evidence of peripheral cholesterol embolization. [3] AERD is part of multisystem disease as embolization often also affects other organs, such as the skin, gastrointestinal system, and brain. [3] The AERD is often is an underdiagnosed clinical illness, due to absence of signs of peripheral embolization or inability to confirm the diagnosis by histology. ${ }^{[1]}$

The release of cholesterol plaques into the circulation can occur spontaneously or after intravascular trauma with angiographic catheters (coronary angiography, coronary angioplasty, aortogram) or after the use of anticoagulants and thrombolytic agents. ${ }^{[1-8]}$ The exact incidence of AERD is not known. ${ }^{[1]}{ }^{[4]}$ The clinical experience is limited to isolated case reports, case series and clinic pathologic case discussions. ${ }^{[3][4][5]}$

\section{AIMS AND OBJECTIVES}

Our objective was to review the clinical profile of patients who were diagnosed as atheroembolic renal disease and to assess their renal function recovery status.

\section{MATERIALS AND METHODS}

All the patients who were referred to and diagnosis as atheroembolic renal disease based on clinical presentation and/or biopsy, were included in the study. This study was conducted in department of Nephrology at Amrita Institute of Medical Sciences and Research Centre, Kochi, Kerala from January 2007 to June 2008.

\section{RESULTS}

There were eleven cases of renal failure (males:10; female:1), aged 55-75 years (Mean: 60; SD :9.5 years) due to AERD, with varying precipitating factors. The precipitating factors were coronary angiography with or without angioplasty in 2 patients, CABG and antiplatelet drugs in 2, thrombolysis along with anticoagulation in 1, antiplatelet agents in 4 and there were no obvious precipitating events in 2 patients.

All the patients with severe acute renal failure (ARF) (eGFR <30 ml/min/1.73m2) in 10 patients and requiring dialysis therapy and the remaining one patient presented with fatigue, loss of appetite, purplish discoloration of toes and a rising serum creatinine level noted during post angioplasty monitoring. Majority (7 out of 11) of patients were nonoliguric at presentation, had uncontrolled hypertension ( 8 out of 11) and microhematuria (9 out of 11). All 11 patients had evidence of peripheral atheroembolism like livodoreticularis and/or purplish discoloration of toes. Two patients had Hollenhorst plaques retina and 4 patients had gangrenous changes in terminal phalanges of toes.

The diagnosis of AERD was confirmed in 3 patients with renal biopsy that showed cholesterol clefts in intrarenal vessels along with mononuclear infiltrates and in others diagnosis was made as they had ARF with evidence of peripheral atheroembolization and had no other cause for renal failure.

Majority (10 out of 11) of patients required renal replacement therapy (RRT). All those requiring RRT were treated with hemodialysis. The predominant indications for hemodialysis were accelerated hypertension with pulmonary edema in 4 , progression to anuria in 4 , hyperkalemia and uremic symptoms in the remaining 2 patients. 
Of the 10 patients who required dialysis 4 patients could discontinue hemodialysis after 2 weeks to 6 months with mild to moderate degree of residual renal dysfunction and remaining 6 are on hemodialysis at the time of study. One among 4 patients who came off hemodialysis, one had worsening of renal functions again and he developed severe renal failure requiring dialysis and he expired after six months on hemodialysis due to a cardiac event. The patient, who did not need dialysis, had partial improvement in renal function with conservative treatment.

\section{DISCUSSION}

Majority of the patients were males (10 out of 11) and most were aged more than 60 years ( 9 out of 11) in our study. The patient profile in terms of male predominance and elderly age is consistent with earlier reports. ${ }^{[5] ~[6]}$ Majority (9 out of 11) of the patients had precipitating factors; coronary angiography with angioplasty and antiplatelet agents in 2 patients; CABG and antiplatelet agents in 2; thrombolysis and antiplatelet agents in 1; and antiplatelet agents alone in 4 subjects. There were no obvious precipitating events in 2 patients. The precipitating factors in our study are similar to earlier reported studies, with only minority of cases being spontaneous AERD. ${ }^{[4][5]}$

Majority (10 out of 11) of the patients in the study presented with had ARF requiring dialysis therapy and the remaining one patient presented with signs of peripheral atheroembolization with fatigue, loss of appetite, purplish discoloration of toes and a rising serum creatinine level noted during post angioplasty monitoring. Acute presentation was seen in majority of patients in our study a finding consistent with an earlier report which had shown that in iatrogenic AERD is more likely to present as acute/subacute renal failure than spontaneous forms of AERD. ${ }^{\text {[2] [4] [5] }}$

In majority (7 out of 11) of patients the ARF was nonoliguric (urine volume $\geq 400 \mathrm{ml} /$ day) at presentation. Majority of the patients had associated uncontrolled hypertension (8 out of 11) and microhematuria (9 out of 11).
Atheroembolization being a systemic phenomenon; evidence of peripheral atheroembolization was present in most of the patients in the form of livodoreticularis; 2 of them had Hollenhorst plaques on funduscopic examination of retina and 4 had gangrenous changes involving terminal phalanges of toes, similar to other studies. ${ }^{[1][2][3][6][7]}$

The diagnosis of AERD could be confirmed in 3 patients with renal biopsy; which showed characteristic features of the disease; cholesterol clefts in intrarenal vessels along with mononuclear infiltrates. ${ }^{[1]}$ In remaining 8 patient's diagnosis of AERD was made as they had ARF with evidence of peripheral atheroembolization and had no other cause for renal failure.

Majority (10 out of 11) of patients required renal replacement therapy (RRT) and were treated with hemodialysis. Only 4 patients had partial recovery of renal function and could discontinue hemodialysis (after 2-24 weeks); however, of the above 4 patients one had worsening of renal functions to severe renal failure requiring dialysis and he expired after six months on hemodialysis due to a sudden cardiac arrest. The poor renal survival in patients with AERD in present study is consistent with earlier reports as the treatment options for the disease are limited. ${ }^{[1-8]}$ Statins and Steroids have been reported to be of benefit by some, and were also administered to our patients. [1] [2] [3]

Given the inability to reverse the condition, early diagnosis is crucial if further episodes of cholesterol emboli are to be prevented. The presence of clinically apparent cholesterol embolization should be considered a contraindication to any further vascular intervention. A highly conservative approach, avoiding anticoagulation, angiography, and vascular surgery may produce the best outcome. Cholesterol embolism should feature prominently in the differential diagnosis of acute renal failure in elderly patients who have undergone thrombolysis, anticoagulation, angiography, or other vascular interventions. 


\section{CONCLUSIONS}

AERD is one of the important cause for ARF in elderly patients; especially those on anticoagulants, antiplatelet medications, those undergoing vascular intervention procedures. The risk of AERD increases especially in those with underlying atherosclerotic vascular disease. Majority of affected patents are males belonging to elderly age. Majority of patients with AERD require RRT. Renal function improves only in minority of patients if they need RRT and time taken for improvement varies between 2-24 weeks. Given the inability to reverse the condition, careful selection of patients for vascular procedures, anticoagulation or thrombolysis is the only way to prevent the occurrence of AERD, as there no effective therapy for this disease.

\section{Source(s) of support: Nil, \\ Conflicts of Interest: Nil}

\section{REFERENCES}

1. Kulwant SM, Venkateswara KR. Atheroembolic Renal Disease. J Am Soc Nephrol 2001; 12: 1781-1787.

2. The Challenge of Diagnosing Atheroembolic Renal Disease: Clinical Features and Prognostic Factors. Circulation2007;116: 298-304. DOI: 10.1161/CIRCULATIONAHA.106.68099 1

3. Scolari F, Ravani P. Atheroembolic renal disease.Lancet. 2010; 375(9726):16501660. DOI: $10.1016 /$ S0140-6736 (09) 62073-0.

4. Granata A, Insalaco M, Di Pietro F, Di Rosa S, Romano G, Scuderi R. Atheroembolism renal disease: diagnosis and etiologic factors. Clin Ter. 2012;163(4):313-322.

5. Elena GS, Enrique M, Angel RJ, F Javier A, Francisco R Carmen V. Atheroembolic renal disease: Analysis of clinical and therapeutic factors that influence its progression. Nefrologia 2010; 30(3):31723. DOI: $10.3265 /$ Nefrologia.pre2010. Apr.10367.

6. Thériault J, Agharazzi M, Dumont M, Pichette V, Ouimet D, Leblanc M, Atheroembolic Renal Failure Requiring Dialysis: Potential for Renal Recovery? A Review of 43 Cases. Nephron ClinPract 2003;94:c11-c18. DOI:10.1159/000070819.

7. McGowan JA, Greenberg A. Cholesterol Atheroembolic Renal Disease: Report of 3 Cases with Emphasis on Diagnosis by Skin Biopsy and Extended Survival. Am J Nephrol 1986; 6:135-139. DOI:10.1159/000167068.

8. Lakshminarayana G, Rajesh R, Seethalekshmy NV, Kurian G, Unni VN. Atheroembolic renal disease following thrombolysis. Indian Journal of Nephrology. 2007; 17 (1): 14-16. 